\title{
TRATAMIENTO POR FETOSCOPIA DEL SÍNDROME DE TRANSFUSIÓN FETO FETAL EN CLÍNICA ALEMANA DE SANTIAGO
}

Masami Yamamoto C. ${ }^{1}$, Julio Astudillo D. ${ }^{1}$, Daniel Pedraza S. ${ }^{1}$, Hernán Muñoz S. ${ }^{1}$, Álvaro Insunza F. ${ }^{1}$, José Fleiderman D. ${ }^{1}$, Rodrigo Riveros $K{ }^{1}$

1 Unidad de Medicina Materno Fetal, Departamento de Ginecología y Obstetricia, Clínica Alemana de Santiago.

\section{RESUMEN}

Antecedentes: El síndrome de transfusión feto fetal (STFF) afecta el 15\% de los gemelares monocoriales y tiene una mortalidad de $90 \%$ cuando se diagnostica antes de las 26 semanas. Objetivo: Evaluar el resultado perinatal mediante fotocoagulación con láser por fetoscopia, de las anastomosis vasculares placentarias en pacientes con STFF. Método: Fotocoagulación láser por fetoscopia de 18 casos de SFF tratados en Clínica Alemana de Santiago entre los años 2005-2008. Resultados: $72,8 \%$ de los embarazos concluyeron con al menos un niño vivo. No se requirió una nueva fetoscopia en ningún caso. No hubo rotura de membranas posprocedimiento en pacientes previamente asintomáticas. No se ha presentado daño neurológico en los niños sobrevivientes. Conclusiones: El resultado materno-perinatal es similar a las series internacionales y demuestra la factibilidad de este procedimiento de ser realizado en nuestro país por profesionales capacitados.

\section{PALABRAS CLAVE: Síndrome de transfusión feto fetal, fetoscopia, embarazo gemelar monocorial, tratamiento intrauterino}

\section{SUMMARY}

Background: Twin to twin transfusion syndrome (TTTS) occurs in $15 \%$ of monochorionic twins and has a $90 \%$ mortality when diagnosed before 26 weeks. Objective: To evaluated the perinatal outcome following fetoscopy guided laser coagulation of placental anatomoses. Method: Retrospective analysis of 18 cases treated in Clinica Alemana, between 2005 and 2008. Results: $72.8 \%$ of the pregnancies had at least one survivor at neonatal discharge. No case needed a second fetoscopy. There was no rupture of the membranes in patients without symptoms before surgery. There were no neurological sequels in survivors. Conclusions: The maternal and perinatal outcome is similar to international series and demonstrates feasibility in our country by experienced operators.

KEY WORDS: Twin to twin transfusion syndrome, fetoscopy, monochonionic twin pregnancy, intrauterine treatment 


\section{INTRODUCCIÓN}

El síndrome de transfusión feto fetal afecta al $15 \%$ de todos los embarazos múltiples monocoriales y tiene la mortalidad perinatal más alta de las patologías obstétricas, superando el $90 \%$ en ausencia de tratamiento. Consiste en la presencia de exceso de líquido amniótico en un feto y ausencia completa en el otro, producto de una descompensación de la distribución sanguínea, progresiva e irreversible, que ocurre en las comunicaciones vasculares placentarias, propias de este tipo de embarazo gemelar.

El tratamiento clásico fue la realización de amniodrenaje a repetición, sin embargo, desde el año 1995 se ha implementado la cirugía por fetoscopia en distintos centros del mundo $(1,2)$, luego del cual se han tratado más de 1000 casos hasta el año 2007 (3). La demostración del beneficio de la fetoscopia y fotocoagulación con láser por sobre el amniodrenaje (4), fue publicado en el año 2004, donde se demostró un aumento de la sobrevida a 6 meses del parto de $51 \%$ en amniodrenajes a $76 \%$ en la fetoscopia-láser, con una sobrevida sin daño neurológico de $31 \%$ en amniodrenajes y $52 \%$ con fetoscopia-láser.

Este hito motivó el desarrollo de la pesquisa precoz de la condición, a fin de evitar las consecuencias letales de la enfermedad como el aborto espontáneo, la prematurez extrema y la muerte fetal. Dentro de este contexto de la necesidad de ofrecer el tratamiento necesario a las pacientes portadoras del diagnóstico, es que se implementó la cirugía fetoscópica.

El objetivo de esta comunicación es presentar el desarrollo y resultados de la implementación de esta técnica en Clínica Alemana de Santiago, entre los años 2005 y 2008.

\section{PACIENTES Y MÉTODO}

Estudio retrospectivo, descriptivo, de una serie de pacientes con diagnóstico de síndrome de transfusión feto fetal (STFF). El diagnóstico antenatal fue basado en la ultrasonografía según los criterios habituales, consistentes en la presencia de polihidroamnios en uno y oligoamnios en otro. Se define como polihidroamnios la presencia de un bolsillo de líquido amniótico (LA) máximo vertical de al menos $8 \mathrm{~cm}$ antes de las 20 semanas y al menos $10 \mathrm{~cm}$ posteriormente. Oligoamnios se define por la ausencia de un bolsillo de LA máximo vertical de más de $2 \mathrm{~cm}$. La fetoscopia se indicó hasta las $26 \mathrm{se-}$ manas, según los criterios actuales. La indicación de la cirugía fue realizada por los tratantes de las pacientes y confirmada en nuestra institución.

La evaluación ecográfica en la Unidad de Medicina Materno Fetal de la Clínica Alemana consistió en la realización de biometría fetal, medición de LA, medición del cuello uterino, Doppler de los territorios arteriales y venosos incluyendo la medición del flujo por la vena umbilical (5).

Procedimiento (6): La cirugía se realizó con las pacientes hospitalizadas. La preparación preoperatoria consistió en indometacina $25 \mathrm{mg}$ cada 6 horas vía oral, desde 12 a 24 horas antes, ducha con povidona jabonosa, diazepam oral $10 \mathrm{mg}$ y cefazolina $1 \mathrm{~g}$ endovenoso preoperatorio. El procedimiento se realizó con técnica aséptica en pabellón quirúrgico. El sitio de inserción del trocar fue decidido previamente en la evaluación ecográfica. Se buscó una inserción no transplacentaria, en que se proyectara una correcta visualización del plato corial (Figura 1). Las pacientes recibieron remifentanil endovenoso. Se utilizó anestesia local con lidocaína al $2 \%$ hasta el miometrio para la inserción del trocar, bajo visión ecográfica evitando los vasos uterinos. Se utilizó un catéter venoso central $10 \mathrm{Fr}$, el cual se insertó hasta la cavidad amniótica por técnica de Seldinger. Se utilizó un Fetoscopio Storz semirrígido, en vaina deformable, de $3,3 \mathrm{~mm}$ de diámetro externo con 2 vías, curvado en casos de placenta anterior. La fuente de energía láser fue YAG en los primeros casos y posteriormente Diodo, con fibras de 600 um. La técnica de la coagulación fue con intención selectiva, es decir, preservando los vasos que cruzan la membrana que se demuestren no ser anastomosis entre los fetos, por el seguimiento hacia los cordones. La Figura 2 muestra una imagen endoscópica de la superficie placentaria con una anastomosis placentaria.

Las pacientes permanecieron hospitalizadas por 2 días y luego dadas de alta para el manejo ambulatorio en sus centros de derivación. En algunos centros de Santiago, las pacientes fueron trasladadas horas después del procedimiento. El seguimiento recomendado fue una ecografía semanal con biometría, Doppler de arteria umbilical, cerebral media para la medición de la velocidad máxima, y medición de cérvix. Se recomendó la interrupción del embarazo a las 35 semanas en forma electiva, o antes en relación a las complicaciones presentes. No se realizó recomendaciones específicas sobre la vía del parto. 




Figura 1. Representación esquemática de un embarazo gemelar monocorial biamniótico con síndrome de transfusión feto fetal, en que la placenta está posterior y la membrana tiene al feto donante en oligoamnios "stuck", adherido, fijo dentro de la cavidad amniótica. El endoscopio se inserta por la piel, y se logra visualizar la superficie corial de la placenta, donde se encuentras las anastomosis. Publicación con permiso AJOG.

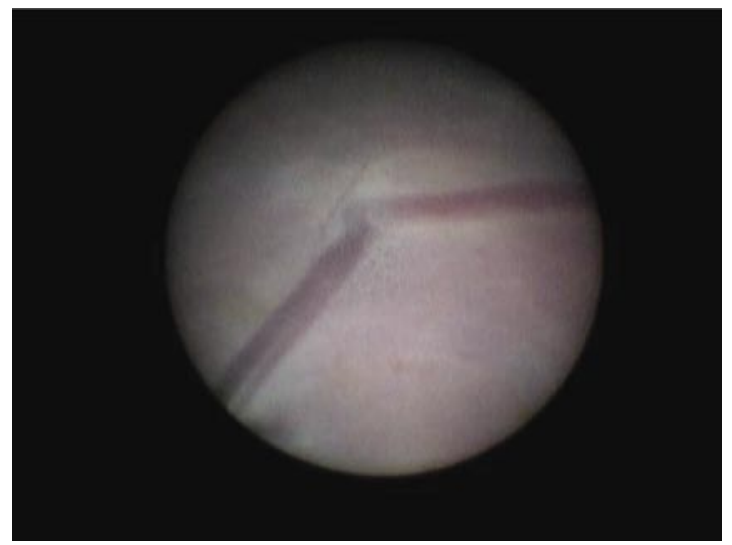

Figura 2. Imagen fetoscópica de una anastomosis arterio-venosa entre un feto donante y un receptor. La arteria viene de la izquierda, demostrada por un color más oscuro, propio de su saturación de oxígeno más baja que la de la vena, a la derecha. Al centro convergen ambos vasos, sumergiéndose en la placenta hacia un cotiledón.

\section{RESULTADOS}

Se trataron 18 casos entre el 2005 y 2008. La Tabla I resume los casos, edad gestacional al momento del tratamiento, la severidad de la enfermedad en estadíos de Quintero (1 a 4), la edad gestacional al parto y el resultado perinatal. En resumen, de los 18 casos tratados: 5 con ambos niños vivos, 8 con un niño vivo, 4 con ambos fetos muertos y 1 con un aborto espontáneo posterior al procedimiento. En esta serie el $72 \%(13 / 18)$ de embarazos terminaron con al menos un niño vivo, con una sobrevida perinatal global de $50 \%$ (18/36). No hay casos con alteración del desarrollo neurológico, aunque esto es todavía precoz pues la mayoría de los niños tienen menos de 2 años.

No hubo complicaciones maternas asociadas al procedimiento. Fue bien tolerado, sin necesidad de utilización de anestesia regional. Las pacientes estuvieron concientes durante el procedimiento.

El caso 1, se trató de un embarazo de 24 semanas con placenta anterior y polihidroamnios sintomático, caracterizado por un aumento importante de la altura uterina y dolor costal. Se realizó fetoscopia con una correcta visualización de la placenta. A las 28 semanas se constató oligoamnios progresivo del feto donante, con flujo ausente en diástole. Se decidió la interrupción del embarazo, con sobrevida de ambos al mes de vida. El feto donante tuvo complicaciones respiratorias, fallece a los 9 meses producto de insuficiencia respiratoria terminal.

El caso 2, se destacó por una dificultad en el procedimiento, en que dada su corta edad gestacional (18 semanas) y el grosor de la pared abdominal materna que impedía una manipulación del endoscopio, no fue posible visualizar el plato corial ubicado en la pared anterior. Este procedimiento fue catalogado como insatisfactorio desde su inicio. La paciente tuvo un postoperatorio caracterizado por dolor abdominal, sin embargo, esto no impidió su traslado por sus medios a su centro de referencia en Concepción, donde posteriormente se constató el óbito de ambos fetos.

El caso 8, tenía un cérvix de $22 \mathrm{~mm}$ previo a la fetoscopia. La paciente había sido informada de tener una contraindicación de fetoscopia en otro centro de Santiago. Definimos que la paciente podía beneficiarse del tratamiento y se realizó la cirugía sin dificultades. Lamentablemente se produjo óbito fetal a las 24 horas, y a los 5 días del segundo. 
Tabla I

\section{RESULTADOS DEL TRATAMIENTO POR FETOSCOPIA DE LOS EMBARAZOS CON SÍNDROME DE TRANSFUSIÓN FETO FETAL}

\begin{tabular}{|c|c|c|c|c|c|c|}
\hline Paciente & $\begin{array}{l}\text { Fecha de } \\
\text { fetoscopia }\end{array}$ & $\begin{array}{l}\text { Semanas al } \\
\text { tratamiento }\end{array}$ & $\begin{array}{l}\text { Estadío } \\
\text { Quintero }\end{array}$ & $\begin{array}{l}\text { Ubicación } \\
\text { placentaria }\end{array}$ & $\begin{array}{l}\text { Semanas } \\
\text { al parto }\end{array}$ & $\begin{array}{l}\text { Resultado } \\
\text { perinatal }\end{array}$ \\
\hline 1 & 01-05-2005 & 23 & 3 & Anterior & 27 & $\begin{array}{l}1 \text { vivo y } \\
1 \text { muerte } \\
\text { infantil }\end{array}$ \\
\hline 2 & 23-06-2006 & 18 & 4 & Anterior & 19 & $\begin{array}{l}\text { Óbito fetal } \\
\text { ambos }\end{array}$ \\
\hline 3 & $15-02-2007$ & 22 & 2 & Anterior & 34 & 2 vivos \\
\hline 4 & $17-02-2007$ & 19 & 3 & Anterior & 35 & $\begin{array}{l}1 \text { vivo y } \\
1 \text { muerte } \\
\text { fetal }\end{array}$ \\
\hline 5 & $30-03-2007$ & 23 & 3 & Lateral & 28 & 2 vivos \\
\hline 6 & 04-02-2008 & 24 & 2 & Posterior & 32 & 2 vivos \\
\hline 7 & $05-02-2008$ & 19 & 2 & Posterior & 28 & 2 vivos \\
\hline 8 & 15-03-2008 & 25 & 2 & Posterior & 25 & $\begin{array}{l}\text { Óbito fetal } \\
\text { ambos }\end{array}$ \\
\hline 9 & $11-04-2008$ & 22 & 3 & Posterior & 23 & $\begin{array}{l}\text { Aborto } \\
\text { espontáneo }\end{array}$ \\
\hline 10 & 14-04-2008 & 23 & 1 & Posterior & 33 & $\begin{array}{l}1 \text { vivo y } \\
1 \text { muerte } \\
\text { fetal }\end{array}$ \\
\hline 11 & $30-04-2008$ & 19 & 2 & Anterior & 27 & $\begin{array}{l}1 \text { vivo y } \\
1 \text { muerte } \\
\text { fetal }\end{array}$ \\
\hline 12 & 14-06-2008 & 23 & 3 & Posterior & 28 & $\begin{array}{l}1 \text { vivo y } \\
1 \text { muerte } \\
\text { fetal }\end{array}$ \\
\hline 13 & $20-06-2008$ & 23 & 1 & Anterior & 34 & $\begin{array}{l}1 \text { vivo y } \\
1 \text { muerte } \\
\text { fetal }\end{array}$ \\
\hline 14 & 20-07-2008 & 21 & 3 & Anterior & 28 & $\begin{array}{l}1 \text { vivo y } \\
1 \text { muerte } \\
\text { fetal }\end{array}$ \\
\hline 15 & $25-10-2008$ & 22 & 3 & Posterior & 35 & 2 vivos \\
\hline 16 & 22-11-2008 & 23 & 3 & Posterior & 29 & $\begin{array}{l}1 \text { vivo y } \\
1 \text { muerte } \\
\text { fetal }\end{array}$ \\
\hline 17 & 04-12-2008 & 18 & 3 & Anterior & 25 & $\begin{array}{l}\text { Óbito fetal } \\
\text { ambos }\end{array}$ \\
\hline 18 & $22-12-2008$ & 19 & 2 & Anterior & 21 & $\begin{array}{l}\text { Óbito fetal } \\
\text { ambos }\end{array}$ \\
\hline
\end{tabular}


El caso 9, tuvo la particularidad de haber presentado contracciones y estar bajo tocólisis y 22 semanas de edad gestacional. Había sido tratada con amniodrenaje previo a la fetoscopia. El cérvix medía $9 \mathrm{~mm}$, con $1 \mathrm{~cm}$ de dilatación y $50 \%$ de borramiento. Se evaluó en conjunto con los médicos tratantes, y se definió que la paciente podría beneficiarse con la fetoscopia, que pudiera tratar definitivamente la transfusión feto fetal, y realizar un drenaje al final, que permitiera la disminución del tamaño uterino y la disminución de las contracciones. Se generó la opinión colegiada que en ausencia de cualquier tipo de tratamiento, el pronóstico del embarazo sería completamente adverso, por lo que en una decisión conjunta con los tratantes y la paciente, se decidió la realización del procedimiento. Este se realizó en conocimiento de una mayor probabilidad de fracaso. Luego del procedimiento no se realizó cerclaje y la paciente pudo ser trasladada a su centro hospitalario con el útero en reposo. Sin embargo progresó con un trabajo de aborto en 24 horas.

El caso 11, fue operado a las 19 semanas y se produjo la muerte del feto donante a las 24 horas del procedimiento. Previo al alta se constató un cérvix de $11 \mathrm{~mm}$ y se realizó cerclaje cervical. El embarazo continuó sin incidentes hasta las 27 semanas, en que se diagnosticó un oligoamnios y Doppler umbilical anormal con flujo ausente en diástole, lo que motivó la interrupción del embarazo. La evolución postnatal fue favorable.

El caso 17, fue diagnosticado precozmente, a las 16 semanas. Desde un inicio tenía anormalidades del Doppler de la arteria umbilical del feto donante, con inserción velamentosa del cordón. Se realizó fetoscopía a las 18 semanas con mejora de las anormalidades del Doppler en el postoperatorio precoz. A las 24 semanas se diagnosticó la presencia de secuencia TAPS (7), consistente en discordancia importante de la velocidad máxima de la arteria cerebral media, con 2,1 MoM (8) en el receptor y $0,5 \mathrm{MoM}$ en el donante. Estos son signos de una anemia en el receptor y policitemia en el donante. En ese momento la estimación de peso fetal era 620 y 450 gramos, respectivamente, con líquido amniótico normal para ambos. Debido a la alta mortalidad neonatal en caso de realizarse una cesárea, se realizó una transfusión intrauterina en el receptor, llevándose el hematocrito de $15 \%$ a $35 \%$, luego de un aporte de $33 \mathrm{ml}$ de sangre. A las 24 horas se constató la muerte del receptor, con signos de insuficiencia cardiaca severa, y al 5ํáa se produjo la muerte del donante.

El caso 18, presentó preeclampsia concomitantemente con el diagnóstico de STFF, con PA
150/90 y proteinuria positiva de 24 horas. La paciente estaba sin contracciones pero con cérvix corto. Se decidió la realización de la fetoscopia en concordancia con la paciente. No hubo incidentes en el procedimiento, y se complementó con cerclaje bajo anestesia peridural. El feto donante fallece a las 24 horas del procedimiento y la paciente fue trasladada a su centro de derivación. Presentó una progresión de la preeclampsia hacia la severidad en 4 días, por lo que se indujo.

Los otros 11 casos tuvieron una evolución postoperatoria esperable para la condición. Ninguna paciente requirió amniodrenajes posterior a la fetoscopia. No se produjo ningún caso de rotura de membranas posprocedimiento en pacientes previamente asintomáticas, por lo que se confirma que el riesgo atribuible al procedimiento es menor al $10 \%$ en esta serie.

Todos los casos pudieron ser operados con anestesia local, sedación y remifentanil endovenoso. Esto permitió evitar descompensaciones hemodinámicas que pudieran hacer necesario el aporte de cristaloides y sus consecuencias. La tolerancia materna al procedimiento fue óptima. Las hospitalizaciones posterior al procedimiento fueron menores a 2 días, salvo en casos de cerclaje.

Dieciséis pacientes eran residentes de Chile, una de Perú y otra de Ecuador. Las pacientes del extranjero viajaron al tercer día del procedimiento. Ocho pacientes provenían del sistema privado y diez del sistema público. Las pacientes del sistema público 5 provenían de Santiago, 2 de Concepción y 1 de lquique, Antofagasta y Valparaíso. Tres pacientes provenientes del sistema público fueron beneficiadas del convenio asistencial entre el Hospital Padre Hurtado y Clínica Alemana de Santiago.

\section{DISCUSIÓN}

El presente estudio muestra el resultado de una serie de casos que han requerido tratamiento intrauterino por STFF. El resultado global es haber podido obtener un $72 \%$ de los casos con al menos un feto vivo, al alta de la unidad de neonatología. Este resultado es comparable con las series internacionales, que han mostrado una tasa de 70 a $90 \%$ que resume más de 1000 casos (9), y similar a otra serie chilena de 33 casos (10). Nuestra serie tiene además la particularidad de haber sido una serie que buscó aportar el beneficio para las pacientes, a pesar de los factores de mal pronóstico que pudieran disminuir las probabilidades de éxito.

Desde el inicio no fue considerado una contraindicación del procedimiento la longitud cervical, la presencia de contractilidad uterina, placenta anterior 
o anomalías del Doppler. De hecho, nuestra población tratada consistió en 9 casos con placenta anterior, 10/18 con anomalías del Doppler, una con preeclampsia concomitante y una con amniodrenaje previo y contracciones. Por este motivo, la sobrevida obtenida en este grupo de mal pronóstico es de gran valor. No debe olvidarse que el mal pronóstico es el fundamento del tratamiento antenatal.

El diagnóstico del STFF, debe realizarse con una ecografía cada 15 días, desde que se realiza el diagnóstico de embarazo gemelar monocorial biamniótico. En cada una de estas ecografías quincenales debe evaluarse el crecimiento, el bolsillo vertical máximo de líquido amniótico y el Doppler de arteria umbilical. La indicación de coagulación láser por fetoscopia se define por la discordancia de líquido amniótico como en todos los centros.

La detección precoz es un concepto interesante que debe desarrollarse en esta oportunidad. Diversas opiniones han surgido para decir que la detección precoz consiste en detectar los casos en etapas 1 ó 2 de Quintero, antes de que se detecten las alteraciones del Doppler o las consecuencias finales de la enfermedad. Esta aseveración tiene errores importantes, pues hasta el momento la clasificación de Quintero (11) no ha podido demostrar ser evolutiva (12), y más bien ha sido considerada como formas diferentes de presentación. Esto no le quita el gran valor de haber permitido estratificar los embarazos en grupos similares. Un estudio retrospectivo de 50 casos de STFF tratados con amniodrenaje (13), demostró que el $22 \%$ de los casos cambió a una etapa menor con solo un amniodrenaje, $40 \%$ se mantuvo y solo el $38 \%$ progresó. Otro estudio prospectivo de 52 casos (14), mostró que entre el 15 a $60 \%$ de los casos mejoró en su clasificación, con una mortalidad similar entre los grupos y con edades gestacionales al diagnótico de 24, 20, 20,21 y 16 semanas para las etapas I a V respectivamente, lo que no corresponde a una progresión. De hecho, la etapa $V$ ha dejado de ser considerada para uso habitual, y se entiende que con la muerte de un feto se detiene el fenómeno transfusional, y evidentemente no se indica el procedimiento.

Entendido que no hay una clara progresión de la enfermedad entre las etapas, el screening precoz debe entenderse como aquel que busca detectar la enfermedad antes de que ella manifieste las complicaciones propias de la historia natural que lleven a la pérdida fetal por aborto u óbito. En este sentido, es ideal poder diagnosticar la condición en ausencia de contracciones o muerte. En nuestro caso, hubo una paciente que fue operada bajo tocolisis, con un amniodrenaje previo, que progresó hacia el aborto espontáneo después del tratamiento por fetoscopia (caso 9).
Otro tema de gran interés es el valor del cerclaje como terapia coadyuvante en el tratamiento del STFF. Luego del amniodrenaje, se produce un relajamiento uterino que disminuye las contracciones, pero también quedan membranas redundantes que pueden herniarse pasivamente por el cérvix. Este procedimiento no es excepcional y existen importantes reportes en la literatura. En una serie de 137 casos de tres centros, donde se estudió el efecto de la longitud cervical preoperatoria (15) y riesgo de aborto y parto, se encontró una asociación entre la longitud del cérvix y estas complicaciones. Nueve de estas pacientes fueron beneficiarias de cerclaje, por tener una longitud menor a $20 \mathrm{~mm}$. El estudio no tiene la capacidad de demostrar el beneficio, sin embargo, confirma la importancia de detectar esta condición que puede tener un potencial beneficio con el cerclaje y su utilización en otros centros.

Luego del tratamiento, los fetos no están libres de complicaciones y no pueden ser considerados como gemelares normales a pesar de la teórica "bicorionización". Ha ocurrido un importante proceso de coagulación placentaria, con necrosis de una parte de ella que puede dejar una distribución placentaria discordante o insuficiente. La muerte de un feto ocurre en cerca del $20 \%$ luego del procedimiento, pudiendo afectar a donantes o receptores. Diversos estudios han buscado detectar factores de riesgo preoperatorios para predecirla $(16,17,18)$.

Las causas de muerte son aún desconocidas para la mayoría de ellas, sin embargo se presume que las principales son la insuficiencia placentaria, la insuficiencia cardiaca, la persistencia de la transfusión feto fetal y la secuencia anemia policitemia (7), Ilamada recientemente "TAPS" por "twin anemia polycythemia sequence". Esta es una complicación producto de una transfusión sanguínea crónica, de pequeño volumen, que produce anemia en un feto y policitemia en el otro, solamente detectable por diferencias en la velocidad máxima de la arteria cerebral media, pues la transfusión no logra producir diferencias de volemia (19). El criterio diagnóstico es la detección de velocidades elevadas persistentes sobre 1,5 MoM en uno, y bajo 0,8 MoM en el otro. Paradójicamente, la anemia se detecta en el feto receptor con mayor frecuencia, y el manejo más apropiado aún está por conocerse. Los casos reportados han sido tratados con transfusión intrauterina seriada en el feto anémico, una nueva fetoscopía para coagular anastomosis persistentes (no coaguladas o repermeabilizadas) o coagulación de cordón de uno de los fetos con el fin de poder salvar uno de ellos (20).

Aunque no hay claridad al respecto, la reoperación es aquella que tiene la mayor racionalidad, en cuanto busca detener la causa etiológica de la 
enfermedad. En nuestra serie, el caso 17 presentó esta complicación, la cual a pesar de las transfusiones intrauterinas no fue posible obtener niños vivos. Lo importante de esto, sin embargo, es que es una complicación que puede aparecer semanas más tarde, y que no se manifiesta en signos ecográficos habituales, como discordancia de líquido amniótico o crecimiento, sino que simplemente por la alteración en el Doppler de la arteria cerebral media. Por este motivo, el seguimiento propuesto para los casos con STFF tratados por fetoscopia consiste en la ecografía semanal hasta el parto, con medición de líquido amniótico, cérvix, biometrías, Doppler de arteria umbilical y arteria cerebral media.

La interrupción electiva deberá realizarse entre las 34 a 35 semanas, ya que en las primeras series se produjo la lamentable muerte fetal a más de 8 semanas del tratamiento. Se explica la muerte fetal por la ocurrencia de insuficiencia placentaria o secuencia TAPS, y posiblemente por la falta de vigilancia para detectar precozmente estas complicaciones. A pesar de la falta de evidencia de esto, la recomendación ha sido aceptada por el Real Colegio de Obstetricia y Ginecología (21). Por otra parte, esta indicación no debe ser considerada tan extraña, pues existen estudios que reportan que los gemelares monocoriales biamnióticos, sin signos ecográficos de transfusión feto fetal, tienen una tasa de muerte fetal entre las 28 y 36 (promedio 34 ) semanas de hasta un $5 \%$ (22), lo que justificaría aún más una interrupción electiva a las 34-35 semanas en casos de STFF tratados por fetoscopia.

\section{CONCLUSIÓN}

Nuestra serie reporta los resultados de un tratamiento altamente complejo, que requiere un proceso diagnóstico en los centros de ecografía del país, una cirugía de alta tecnología y un seguimiento a distancia no menos complejo. A pesar de ello, nuestros resultados son adecuados y han podido aplicarse sin diferencia a una población de distintos sistemas previsionales. El contacto directo con los médicos tratantes ha permitido apoyar las decisiones de interrupción y tratamientos posteriores. La serie publicada demuestra la factibilidad del procedimiento y resultados de nivel comparable de las series internacionales y seguridad para nuestra población.

\section{BIBLIOGRAFÍA}

1. Ville $\mathrm{Y}$, Hyett $\mathrm{J}$, Hecher $\mathrm{K}$, Nicolaides K. Preliminary experience with endoscopic laser surgery for severe twin-twin transfusion syndrome. New Engl J Med 1995;332:224-7.
2. De Lia, J, Kuhlmann R, Harstad T, Cruikshank D. Fetoscopic laser ablation of placental vessels in severe previable twin-twin transfusion syndrome. Am J Obstet Gynecol 1995;172(4),1202-11.

3. Yamamoto $M$, Ville $Y$. Laser treatment in Twin to Twin transfusion syndrome. Sem Fetal Neonat Med 2007;12:450-7.

4. Senat MV, Deprest J, Boulvain M, et al. Endoscopic laser surgery vs serial amnioreduction for severe twin-to-twin transfusion syndrome. New Engl J Med 2004;351:136-44.

5. Yamamoto M, Nasr B, Ortqvist L, Bernard JP, Takahashi Y, Ville Y. Intertwin discordance in the umbilical vein volume flow. A reflection of blood volume imbalance in twin-to-twin transfusion syndrome. Ultrasound Obstet Gynecol 2007;29:317-20.

6. Yamamoto M, El Murr L, Robyr R, Leleu F, Takahashi $\mathrm{Y}$, Ville $\mathrm{Y}$. Incidence and impact of perioperative complications in 175 fetoscopy-guided laser coagulations of chorionic plate anastomoses in fetofetal transfusion syndrome before 26 weeks of gestation. Am J Obstet Gynecol 2005;193(3 Pt 2):1110-6.

7. Robyr R, Lewi L, Salomon L, Yamamoto M, Bernard $\mathrm{J}-\mathrm{P}$, Deprest I, Ville Y Prevalence and management of late fetal complications following successful selective laser coagulation of chorionic plate anastomoses in twin-to-twin transfusion syndrome. Am J Obstet Gynecol 2006;194(3):796-803.

8. Mari G, Deter RL, Carpenter RL, Rahman F, Zimmerman R, Moise KJ Jr, Dorman KF, Ludomirsky A,Gonzalez R, Gomez R, Oz U, Detti L, Copel JA, Bahado-Singh R, Berry S, Martinez- Poyer J, Blackwell SC. Nonivasive diagnosis by Doppler ultrasonography of fetal anemia due to maternal red-cell alloimmunization: collaborative group for Doppler assessment of the blood velocity in anemic fetuses. $\mathrm{N}$ Engl $\mathrm{J}$ Med 2000;342:9-14.

9. Yamamoto $M$, Ville $Y$. Laser treatment in Twin to Twin transfusion syndrome. Sem Fetal Neonat Med 2007;12:450-7.

10. Sepulveda W, Wong A, Dezerega V, Devoto J, Alcalde JL. Endoscopic laser surgery in severe second-trimester twin-twin transfusión síndrome: a three year experience from a latin american center. Prenat Diagn 2007;27:1033-8.

11. Quintero RA, Morales WJ, Allen MH, Bornick PW, Johnson PK, Kruger M. Staging of twin-twin transfusion syndrome. J Perinatol 1999;19:550-5.

12. Luks F, Carr S, Plevyak M, Craigo S, Athankassiou A, Ralston S, Tracy T. Limited prognostic value of a staging system for Twin-to-Twin transfusion syndrome. Fetal Diagn Ther 2004;19:301-4.

13. Cincotta R, Chan FY, Cuncombe G, et al. A staged assessment of the progression of twin-twin transfusion syndrome (TTTS). Am J Obstet Gynecol 2003; S224: 604.

14. Taylor MJ, Govender L, Wee L, et al. Validation of the Quintero staging system for twin-twin transfusion syndrome. Obstet Gynecol 2002;100:1257-65.

15. Robyr R, Boulvain M, Lewi L, Huber A, Hecher K, Deprest $\mathrm{J}$, Ville Y. Cervical Length as a prognostic factor for preterm delivery in twin-to-twin transfusion syndrome treated by 
fetoscopic laser coagulation of chorionic plate anastomoses. Ultrasound Obstet Gynecol 2005;25:37-41.

16. Cavicchioni $O$, Yamamoto $M$, Robyr R, Takahashi $Y$ Ville $\mathrm{Y}$. Intrauterine fetal demise following laser treatment in twin-to-twin transfusion syndrome. BJOG 2006;113(5):590-4.

17. Taylor M, Denbow M, Duncan K, Overton T, Fisk $\mathrm{N}$. Antenatal factors that predict outcome in twintwin transfusion syndrome. Am J Obstet Gynecol 2000;183:1023-8.

18. Martínez JM, Bermúdez C , Becerra C, López J, Morales WJ, Quintero RA. The role of Doppler studies in predicting individual intrauterine fetal demise after laser therapy for twin-twin transfusion syndrome. UItrasound Obstet Gynecol 2003;22:246-51.

19. Mari G, Deter RL, Carpenter RL, Rahman F, Zimmerman R, Moise KJ Jr., et al. Nonivasive diagnosis by
Doppler ultrasonography of fetal anemia due to maternal red-cell alloimmunization: collaborative group for Doppler assessment of the blood velocity in anemic fetuses. N Engl J Med 2000;342:9-14.

20. Yamamoto M, Ville $Y$. Recent findings on laser treatment of twin-to-twin transfusion syndrome. Curr Opin Obstet Gynecol 2006;18:87-92.

21. Yamamoto M, Ville $Y$. Chapter 13. Twin-to-Twin Transfusion Syndrome. En: Multiple Pregnancy: Proceedings of the 50th Royal College of Obstetrics and Gynaecology Study Group. 2006.

22. Barigye O, Pasquini L, Galea P, Chambers H, Chappell L, Fisk N. High Risk of unexpected late fetal death in monochorionic twins despite intensive ultrasound surveillance: A cohort study. PLoS Med 2005;2(6): e172. 